\title{
Correction to: A Life Course Perspective on the Ways Older Men and Women Discuss Sexual Issues
}

\author{
Liat Ayalon $^{1}$ (1) $\cdot$ Inbar Levkovich ${ }^{2} \cdot$ Ateret Gewirtz-Meydan $^{3,4} \cdot$ Khaled Karkabi $^{5}$
}

Published online: 30 October 2018

○) Springer Science+Business Media, LLC, part of Springer Nature 2018

\section{Correction to: Archives of Sexual Behavior} https://doi.org/10.1007/s10508-018-1255-y

The surname of co-author Inbar Levkovich was misspelled (as "Levkovitz") in this originally published article. The original article has been corrected. The corresponding author apologizes for not detecting this error during the proof correction phase.

The original article can be found online at https://doi.org/10.1007/ s10508-018-1255-y.

Liat Ayalon

liat.ayalon@biu.ac.il

1 The Louis and Gabi Weisfeld School of Social Work, Bar-

Ilan University, 52900 Ramat Gan, Israel

2 The Division of Family Medicine, The Ruth and Bruce Rappaport Faculty of Medicine, Technion-Israel Institute of Technology, Haifa, Israel

3 Department of Sociology, Crimes Against Children Research Center and Family Research Laboratory, University of New Hampshire, Durham, NH, USA

4 Sex and Couples Therapy Unit, Meir Medical Center, Kfar-Saba, Israel

5 Department of Family Medicine, The Ruth and Bruce Rappaport Faculty of Medicine, Technion-Israel Institute of Technology, Clalit Health Services, Haifa and Western Galilee District, Israel 